\title{
Interaktionsarbeit als wichtige Arbeitstätigkeit im Dienstleistungssektor
}

Nach einer Untersuchung von ver.di haben drei Viertel der Beschäftigten im Dienstleistungsbereich häufig mit Kunden, Klienten und Patienten zu tun (ver.di 2011). Die besonderen Anforderungen und Unterschiede dieser Arbeit zur Arbeit mit materiellen und immateriellen Objekten werden erst in der neueren Entwicklung in der Arbeitsforschung und Arbeitspolitik systematisch beachtet. Das Konzept der Interaktionsarbeit bietet hierfür eine Grundlage und eröffnet neue Perspektiven in der Auseinandersetzung mit Dienstleistungsarbeit.

\section{Anstöße}

Bei der Arbeit mit Kunden, Klienten und Patienten ${ }^{1}$ bestehen Anforderungen, die bei sonstiger Arbeit nicht in gleicher Weise auftreten. Wichtige Anstöße zur systematischen Bestimmung solcher Besonderheiten ergaben sich zunächst eher an den Rändern der Arbeitsforschung. So wurde in sozialpolitisch orientierten Untersuchungen zu sozialen Diensten die „Ko-Produktion“ herausgestellt (Badura/Gross 1976). Sie bezieht sich darauf, dass Kunden und Klienten nicht als passive Konsumenten auftreten, sondern als Ko-Akteure an der Erstellung der Dienstleistung mitwirken. Am Beispiel von Stewardessen im Flugverkehr wurde auf besondere Anforderungen an das emotionale Verhalten bei Service und Betreuung von Passagieren aufmerksam gemacht (Hochschild 1979), und Untersuchungen im Gesundheitsbereich verwiesen darauf, dass die Beeinflussung der Gefühle von $\mathrm{Pa}$ tienten sowohl für deren Zufriedenheit als auch das Gelingen der medizinischen Behandlung eine wichtige Rolle spielt (Strauss et al. 1980). ${ }^{2}$ In den 1990er Jahren entsteht vor diesem Hintergrund eine neue Phase der Beachtung der Arbeit mit Kunden und Klienten in der Arbeitsforschung. In dem 1999 erschienenen Themenheft „Personenbezogene Dienstleistung - Arbeit der Zukunft" der Zeitschrift für Arbeitswissenschaft wird das Konzept der Interaktionsarbeit vorgestellt und damit die soziale Interaktion als Kern der Arbeit mit Kunden und Klienten bestimmt. ${ }^{3}$ Ausgangspunkt des Konzepts der Interaktionsarbeit ${ }^{4}$ ist die Einsicht, dass sich das in der Gesellschaft und in der Arbeitsforschung vorherrschende Verständnis von Arbeit auf die Arbeit mit materiellen und immateriellen Objek- ten bezieht und dabei das Ziel der Naturbeherrschung zugrunde liegt (vgl. Ernst/ Kopp 2011, S. 262). ${ }^{5}$ Die Übertragung auf die Arbeit mit und an Menschen beinhaltet die Gefahr, dass Kunden und Klienten wie Objekte gesehen werden und ihre Bedürfnisse wie auch Eigenaktivitäten als Störfaktoren erscheinen, die es möglichst auszuschalten gilt. Das Konzept der Interaktionsarbeit begreift demgegenüber den Menschen als Subjekt, gerade auch dann, wenn er zum Gegenstand von Arbeit wird . Damit bezieht es sich auch darauf, dass die Arbeit mit Kunden und Klienten in hohem Maße Unbestimmtheiten und Unwägbarkeiten aufweist. Diese resultieren daraus, dass Kunden und Klienten eigene Interessen und Bedürfnisse haben, selbstständig handeln und nicht unmittelbar der Organisation von Dienstleistungsprozessen unterliegen. So zeigen empirische Untersuchungen, die den Blick auf die alltägliche Arbeitspraxis richten, dass auch bei hoch standardisiertem Kontakt zu Kunden und Klienten, wie bspw. bei Callcenter-Arbeit, Unbestimmtheiten auftreten, die nur durch ein selbstreguliertes, situatives Handeln der Beschäftigten bewältigt werden können (vgl. Holtgrewe 2001; Kleemann/ Matuschek 2003).

Bei der Interaktionsarbeit ergeben sich sowohl besondere Anforderungen wie auch Belastungen. Dies hat auch Konsequenzen für die Arbeitsgestaltung und Arbeitspolitik. Es ergeben sich hieraus sowohl (sachliche) Grenzen für die tayloristische Rationalisierung als auch besondere Ambivalenzen selbstverantwortlicher Arbeit sowie auch neue Kriterien für die Beurteilung beruflicher Qualifikationen und Kompetenzen. Im Folgenden werden zunächst die Merkmale der Interaktionsarbeit ausführlicher dargestellt (Abschnitt 3) und daran anschließend einige arbeitspolitische Konsequenzen umrissen (4). Da das Konzept der Interak- tionsarbeit leicht zu Missverständnissen in seinem Geltungsbereich führt, gehe ich hierauf zuvor noch kurz ein (2).

\section{Geltungsbereich}

Bei der Beschreibung und Klassifizierung von Dienstleistungen wird zumeist

\footnotetext{
1 Im Folgenden wird wegen der Lesbarkeit nur Kunden und Klienten verwendet.

2 Siehe als Überblick über die wissenschaftliche Diskussion bis Ende der 1990er Jahre ausführlicher Dunkel/Weihrich (2010).

3 Hinweise hierauf finden sich auch bereits bei Nerdinger (1994).

4 In der wissenschaftlichen Diskussion wird anstelle des Begriffs der Interaktionsarbeit auch der Begriff der interaktiven Arbeit (Dunkel/ Weihrich 2010) oder der dialogisch-interaktiven Erwerbsarbeit (Hacker 2009) verwendet. Dabei werden einzelne Aspekte der Arbeit mit Kunden und Klienten herausgestellt oder die Arbeitstätigkeit wird insgesamt in ihren personenbezogenen und objektbezogenen Inhalten erfasst. Das Konzept der Interaktionsarbeit konzentriert sich demgegenüber auf die soziale Interaktion und verbindet unterschiedliche hierauf ausgerichtete Forschungsansätze (vgl. auch Ernst/Kopp 2011).

5 Siehe zum vorherrschenden Verständnis von Arbeit als instrumentell-gegenstandsbezogenes und planmäßig-rationales Handeln ausführlicher Böhle (2010) sowie grundlegend auch Hacker (2005).
}

Fritz Böhle, Dr., ist Professor für Soziologie und Leiter der Forschungsstelle Sozioökonomie der Arbeits- und Berufswelt an der Universität Augsburg sowie Vorsitzender des Vorstands des Instituts für Sozialwissenschaftliche Forschung e. V. (ISF) München. Arbeitsschwerpunkte: Verwissenschaftlichung von Arbeit und Erfahrungswissen, subjektivierendes Arbeitshandeln und informelle Organisation. e-mail: fritz.boehle@phil.uni-augsburg.de; fritz.boehle@isf-muenchen.de 
zwischen personenbezogenen Dienstleistungen wie Gesundheitsversorgung oder Erziehung und sachbezogenen Dienstleistungen wie Handel oder Versicherungen unterschieden. Bei personenbezogener Dienstleistung ist der Arbeitsgegenstand kein materielles oder immaterielles Objekt, sondern ein Subjekt. Die Arbeit mit und an Menschen beschränkt sich jedoch nicht nur auf soziale Dienste u. ä., sondern ist ein Merkmal nahezu jeder Dienstleistung. Sie findet an der Schnittstelle zu Kunden und Klienten statt - in der Pflege und Erziehung ebenso wie im Banken- und Versicherungsgeschäft, im Handel, im Callcenter oder bei der Softwareentwicklung. Dementsprechend wird in der neueren Dienstleistungsforschung zwischen Backoffice und Frontoffice oder Frontlinework unterschieden (Frenkel et al. 1999). Ein Merkmal der Frontlinework ist der Kontakt zu Kunden und Klienten. Dieser kann ein Kern der Arbeitstätigkeit sein wie beispielsweise bei der Beratung und Pflege, oder ergänzend bei sachbezogenen Aufgaben auftreten wie im technischen Service.

Damit geraten Ähnlichkeiten zwischen unterschiedlichen Arbeitstätigkeiten und Berufen in den Blick, die bei herkömmlichen Klassifikationen kaum aufscheinen: so z. B. Ähnlichkeiten zwischen der Arbeit bei kundenorientierter Softwareentwicklung, der Beratung im Versicherungsgeschäft, dem Verkauf im Handel und der Pflege oder Erziehung. ${ }^{6}$ Das Konzept der Interaktionsarbeit beleuchtet dabei vor allem das Arbeitshandeln und damit das „wie“ des Arbeitens. Dies erlangt, je nach bestehenden Arbeitsaufgaben und Anforderungen („was“), eine jeweils spezifische inhaltliche Ausformung.

Die standardisierte Arbeit im Callcenter ebenso wie der Bankautomat oder auch die Verlagerung auf die Arbeit des Kunden (Voss/Rieder 2005) erscheinen jedoch leicht als ein Beleg dafür, dass Dienstleistungen auch ohne Interaktionsarbeit möglich sind. Interaktionsarbeit erscheint in dieser Sicht als ein Auslaufmodell oder/und nur auf bestimmte, eingegrenzte Bereiche von Dienstleistungen beschränkt. Ausgeblendet und unterschätzt werden dabei aber die besonderen Leistungen der Interaktionsarbeit. Diese bestehen - wie schon erwähnt - vor allem in der Berücksichtigung der Individualität und Subjektivität von Kunden und Klienten und der damit verbundenen Bewältigung von Unbestimmtheiten. Dies wiederum ist eine wesentliche Vorausset- zung für die Qualität von Dienstleistungen und der viel zitierten Kundenorientierung als Grundlage für die Erschließung neuer Märkte. So wird nicht nur in den Kernsektoren der Dienstleistungen, sondern auch bei der Güterproduktion die Interaktion mit Kunden etwa durch Beratung oder bei der Bearbeitung von Problemlösungen als eine Leistung erkannt, durch die Produkte "veredelt" und neue Märkte erschlossen werden können (Heidling et al. 2010; Baethge/Willkens 2001; Spath/Ganz 2009, Kap II). Obwohl sich Interaktionsarbeit mit unterschiedlichem Gewicht - bei nahezu sämtlichen Dienstleistungen findet, ist Dienstleistungsarbeit aber nicht nur Interaktionsarbeit. Dies wurde bereits mit der Unterscheidung von Backoffice und Frontlinework deutlich. Nicht nur Sachbearbeiter in Versicherungen, sondern auch Angestellte in Verlagen und Presse haben zu großen Teilen keinen unmittelbaren Kontakt mit Kunden und Klienten. Und auch die Frontlinework ist nicht per se identisch mit Interaktionsarbeit - auch dann, wenn die Arbeit mit Kunden und Klienten die Kernaufgabe ist (vgl. Hacker 2005, 2009). So finden etwa auch bei der Pflege Tätigkeiten statt, die sich auf materielle und immaterielle Objekte richten, wie Dokumentation und die Vorbereitung von Arzneien wie auch die Reinigung des Zimmers oder auch das Wechseln der Bettwäsche von Patienten. Das Konzept der Interaktionsarbeit richtet sich demgegenüber ausschließlich auf den Kontakt zu Kunden und Klienten und berücksichtigt die Arbeit mit materiellen und immateriellen Objekten nur so weit, als diese als Hilfsmittel dienen.

\section{Merkmale von Inter- aktionsarbeit}

Das im Folgenden skizzierte Konzept der Interaktionsarbeit greift unterschiedliche Forschungsansätze auf und verbindet sie. ${ }^{7}$

Ein grundlegendes Merkmal von Interaktionsarbeit ist, dass hierbei die soziale Interaktion Arbeit ist. Dies ist zu betonen, da in den Sozialwissenschaften zumeist die soziale Interaktion dem zweckgerichteten, instrumentellen Arbeitshandeln gegenübergestellt wird. Bei der Interaktionsarbeit ist demgegenüber die soziale Interaktion der zentrale In- halt der Arbeit und richtet sich darauf, ein bestimmtes Ergebnis zu erzielen. Sie hat damit eine instrumentelle, zweckgerichtete Ausrichtung. ${ }^{8}$ Dies beinhaltet auch, dass Interaktionsarbeit eine Reihe von Gemeinsamkeiten mit sonstiger Arbeit hat. Sie kann, ebenso wie sonstige Arbeit, betriebsförmig, als Erwerbsarbeit und abhängige Beschäftigung oder selbstständige Beschäftigung organisiert werden und wird in ihrer konkreten Ausprägung durch die Arbeitsorganisation und technische Arbeitsmittel bestimmt. Das Konzept der Interaktionsarbeit betont dabei allgemeine Besonderheiten und Unterschiede des Arbeitshandelns zur sonstigen Arbeit. Arbeitsorganisation und Technisierung werden (zunächst) nicht einbezogen. Jedoch erfolgt die Bestimmung der Besonderheiten von Interaktionsarbeit mit der Absicht, nicht nur besondere Anforderungen an die Arbeitenden, sondern ebenso besondere Anforderungen an die Arbeitsgestaltung in den Blick zu rücken (vgl. Abschnitt 4). Die im Folgenden näher erläuterten Merkmale der Interaktionsarbeit sind: die wechselseitige Abstimmung unterschiedlicher Interessen, der Einfluss auf die Gefühle anderer, der Umgang mit eigenen Emotionen und das subjektivierende Handeln sowie die Kontrolle durch Kunden.

\subsection{WECHSELSEITIGE ABSTIMMUNG VON INTERESSEN ${ }^{9}$}

Da Kunden und Klienten als Ko-Akteure bei der Erstellung von Dienstleistungen auftreten, ist grundsätzlich eine wechselseitige Abstimmung von Interessen notwendig. Die Interessen und Vorstellungen der Dienstleistenden einerseits und der

6 In der wissenschaftlichen Diskussion wird daher auch vorgeschlagen, die Bezeichnung "personenbezogene Dienstleistung " nicht auf eine besondere Dienstleistungssparte zu beziehen, sondern auf den Kontakt zu Kunden und Klienten bei - im Prinzip - sämtlichen Dienstleistungen (vgl. Böhle/ Glaser 2006, S. 12f.).

7 Grundlegend hierfür ist das von Büssing et al. entwickelte integrierte Konzept der Interaktionsarbeit (vgl. Böhle/Glaser 2006). Dabei wird u. a. eine Systematisierung der Rolle von Gefühlen in der Dienstleistungsarbeit aufgegriffen, die bereits schon früher von Dunkel (1988) vorgeschlagen wurde.

8 In der wissenschaftlichen Diskussion wird daher unter Bezug auf den instrumentellen Charakter der sozialen Interaktion bei Dienstleistungsarbeit diese auch als ein strategisches Handeln bezeichnet (Dunkel/Weihrich 2006, S. 70f.). 
Kunden und Klienten andererseits sind weder per se deckungsgleich noch komplementär. Dies bezieht sich sowohl auf die Festlegung des Arbeitsergebnisses als auch auf die Art und Weise, wie es erreicht wird. So sind sich Kunden und Klienten oft nicht im Klaren darüber, was sie brauchen oder können dies schwer exakt definieren. Die Definition des zu erzielenden Ergebnisses ist daher selbst ein Inhalt der Dienstleistungsarbeit. Die notwendige wechselseitige Abstimmung ist eine höchst voraussetzungsvolle Angelegenheit. Sie wird besonders deutlich, wenn von Kunden und Klienten Forderungen geltend gemacht werden, die im Rahmen der verfügbaren personellen, sachlichen und zeitlichen Ressourcen nicht zu realisieren sind, oder Kunden und Klienten sich der Realisierung einer Dienstleistung widersetzen und damit die Dienstleistenden bei ihrer Arbeit eher behindern als fördern.

\subsection{EINFLUSS AUF GEFÜHLE ANDERER ${ }^{10}$}

Eine weitere Besonderheit der Interaktionsarbeit ist der Einfluss auf die Gefühle von Kunden und Klienten. Das Arbeitshandeln beeinflusst neben den funktional-sachlichen Wirkungen auch die emotionale Befindlichkeit von Kunden und Klienten. Hierdurch wird sowohl das Ergebnis der Dienstleistung als auch deren Herstellung beeinflusst. Es sind daher besondere Aktivitäten notwendig, um auch eine für das Gelingen der Dienstleistung förderliche emotionale Verfassung von Kunden und Klienten zu gewährleisten. Diese reicht von der expliziten Einhaltung von meist für selbstverständlich erachteten Umgangsformen und Erzeugung von Vertrauen bis hin zur Einfühlung in besondere Problemlagen, der bewussten Zurückhaltung belastender Informationen und nicht zuletzt dem äußeren Erscheinungsbild. Eine solche Gefühlsarbeit als Teil der Interaktionsarbeit hat wesentlichen Einfluss auf die Qualität der Dienstleistung, sie beinhaltet aber auch die Gefahr, dass Kunden und Klienten manipuliert und sachlich-funktionale Mängel der Dienstleistung verschleiert werden. Das Konzept der Interaktionsarbeit macht damit nicht nur auf Voraussetzungen für das Gelingen der Dienstleistung aufmerksam, sondern auch auf besondere Risiken und Probleme der Dienstleistungsarbeit sowohl für die Arbeitenden als auch für die Kunden und
Klienten. Dies gilt auch für den Umgang mit den eigenen Gefühlen.

\subsection{UMGANG MIT EIGENEN GEFÜHLEN ${ }^{11}$}

Der Umgang mit den eigenen Gefühlen ist keine Besonderheit der Interaktionsarbeit. Die Kontrolle der eigenen Emotionen zählt zu grundlegenden Merkmalen eines zweckund zielgerichteten Arbeitshandelns. Bei der Interaktionsarbeit wirkt sich jedoch die emotionale Verfassung der Arbeitenden nicht nur auf das Arbeitshandeln aus, sondern hiervon unabhängig auch auf das Ergebnis. Dementsprechend bestehen implizite wie auch explizite Anforderungen an ein auf die Wünsche und Bedürfnisse von Kunden und Klienten ausgerichtetes emotionales Verhalten. In den Forschungen hierzu wird unterschieden zwischen der oberflächlichen Demonstration des erwarteten emotionalen Verhaltens einerseits und der Anpassung des eigenen Empfindens hieran andererseits. In Letzterem wurde zunächst eine besondere Belastung wie auch Gefahr der Entfremdung gesehen. In der aktuellen Diskussion wird dies auf der Grundlage empirischer Befunde zwar als eine mögliche, nicht aber als eine zwingende Folge betrachtet.

\subsection{SUBJEKTIVIERENDES HANDELN ${ }^{12}$}

Sowohl der Einfluss auf Gefühle von Kunden und Klienten als auch der Umgang mit eigenen Emotionen richtet sich nicht auf den sachlich-funktionalen Kern der Dienstleistung, sondern ergänzt diesen. Dementsprechend wird bspw. in Untersuchungen hierzu unterschieden zwischen dem Einfluss auf die Gefühle von Kunden und Klienten einerseits und der Hauptarbeitsaufgabe wie medizinische Versorgung u. ä. Ursprünglich und teils auch noch gegenwärtig wird bei der Hauptarbeitsaufgabe kein Unterschied zu sonstiger Arbeit gesehen. ${ }^{13}$ Das Konzept der Interaktionsarbeit bezieht sich demgegenüber gerade auch hier auf Besonderheiten der Arbeit mit und an Menschen.

Bereits weiter oben wurde schon darauf hingewiesen, dass die Arbeit mit und an Menschen durch eine strukturelle Unbestimmtheit geprägt ist. Die Dienstleistung kann daher nur dann erbracht werden, wenn die Arbeitenden in der Lage sind, Unvorhersehbarkeiten und Unwägbarkeiten zu bewältigen. Ein plan- mäßig-zielorientiertes Handeln nach dem Grundsatz „erst denken/entscheiden und dann handeln“ ist hierfür nur begrenzt geeignet. Notwendig ist ein situatives Handeln, durch das auf Unvorhersehbares und auf variierende Situationen unmittelbar reagiert werden kann und auch ohne vorhergehende Planung Arbeitsaufgaben bewältigt werden. In der Arbeitsforschung wird hier unterschieden zwischen einem planmäßig-objektivierenden und erfahrungsgeleitet-subjektivierenden Handeln. ${ }^{14}$ Die Vorgehensweise ist beim subjektivierenden Handeln im Unterschied zu einem planmäßigen Vorgehen dialogischexplorativ. Planung und Ausführung sind nicht voneinander isoliert und verlaufen sequenziell, sondern sind wechselseitig miteinander verschränkt. Durch und im praktischen Handeln werden Lösungswege eruiert und Ziele modifiziert, wie auch (erst) konkret festgelegt. Dies ist verbunden mit einer sinnlichen Wahrnehmung, die sich nicht nur auf exakt wahrnehmbare und eindeutig definierbare Informationen

9 Siehe zum Merkmal der wechselseitigen Abstimmung von Interessen bei der Arbeit mit Kunden und Klienten Weihrich/Dunkel (2003); Dunkel/ Voss (2004) sowie Dunkel/Weihrich (2006)

10 Siehe grundlegend zur Gefühlsarbeit als Bestandteil der Interaktionsarbeit Strauss et al. (1980) sowie den Überblick über weitere Forschungsarbeiten hierzu bei Giesenbauer/Glaser (2006, S. 68ff.).

11 Siehe grundlegend zu Emotionsarbeit als Teil der Interaktionsarbeit Hochschild (1983) sowie den Überblick der hierzu vorliegenden und weiterführenden Forschungen bei Giesenbauer/Glaser (2006, S. 62f.) und Zapf (2002) sowie zu aktuellen Forschungen in: Praeview - Zeitschrift für innovative Arbeitsgestaltung und Prävention 2010 (3).

12 Siehe ausführlicher zu subjektivierendem Arbeitshandeln allgemein bei Böhle et al. (2004) sowie die hierzu vorliegenden empirischen Untersuchungen von Dienstleistungen (Böhle 1999; Weishaupt 2006; Weishaupt et al. 2006; Dunkel 2006; Koch 2010).

13 In dieser Sicht besteht kein grundlegender Unterschied des Arbeitshandelns bei der Verabreichung einer Spritze in der Gesundheitsversorgung und der Bearbeitung eines Werkstücks in der Metallbearbeitung (vgl. Strauss et al. 1980, S. 629; Brucks 1999).

14 Das subjektivierende Handeln findet sich auch in anderen Arbeitsbereichen; es hat jedoch bei Interaktionsarbeit einen anderen Stellenwert. Beim subjektivierenden Handeln wird der Arbeitsgegenstand grundsätzlich "wie“ bzw. „als" ein Subjekt wahrgenommen. Bei sonstiger Arbeit bezieht sich dies auf in der Praxis auftretende Grenzen der wissenschaftlich-technischen Beherrschung und Berechenbarkeit (auch) materieller und immaterieller Objekte, die in solchen Situationen "wie" etwas Lebendiges erscheinen. Bei Interaktionsarbeit bezieht sich dies demgegenüber auf das zentrale Merkmal des Arbeitsgegenstandes. 
richtet. Handlungsleitend ist eine spürende und empfindende Wahrnehmung, die auch diffuse Informationsquellen wie Körper und Gesichtsausdruck oder Atmosphären und Stimmungen aufnimmt und zur Orientierung nutzt. Damit korrespondiert ein das praktische Handeln begleitendes und mitlaufendes sowie bildhaftes und assoziatives Denken. Eine Voraussetzung wie auch Folge des explorativ-dialogischen Vorgehens und der spürenden empfindenden Wahrnehmung ist eine Beziehung zum Arbeitsgegenstand, die nicht auf Distanz, sondern auf Nähe und Verbundenheit beruht.

\subsection{KONTROLLE DURCH DEN KUNDEN ${ }^{15}$}

Und schließlich sind bei der Interaktionsarbeit Kunden und Klienten nicht nur Ko-Akteure, die bei der Erstellung der Dienstleistung mitwirken, sondern sie beurteilen und kontrollieren auch die Dienstleistenden. Bei abhängiger Beschäftigung unterliegen damit die Arbeitenden einer doppelten Kontrolle ihrer Arbeit. Dabei kann Beurteilung durch Kunden und Klienten auch gezielt von den Unternehmen genutzt werden. Für die Arbeitenden kann sich damit eine besondere Anerkennung ihrer Arbeit verbinden, aber auch besondere Belastungen und Restriktionen.

\section{1}

\section{Konsequenzen für die Arbeitspolitik}

Begreift man die Arbeit mit Kunden und Klienten als Interaktionsarbeit, so ergeben sich neue Anstöße und Herausforderungen für die Arbeitspolitik - für die Gestaltung der Arbeitsorganisation ebenso wie für die Leistungsbeurteilung und die berufliche Bildung. Praktiken, die bisher eher vereinzelt und lediglich als Ergänzung $\mathrm{zu}$ vorherrschenden Standards teils auch informell entwickelt wurden, ${ }^{16}$ erhalten damit eine systematische Grundlage und Perspektive für die weitere Entwicklung.

Die wissenschaftliche Diskussion richtet sich bisher allerdings vor allem auf die besonderen Anforderungen bei Interaktionsarbeit und damit verbundenen Belastungen. Im Mittelpunkt stehen dabei psychoemotionale Belastungen. ${ }^{17}$ Untersuchungen zur Vermeidung solcher
Belastungen richten sich bisher primär auf das Verhalten der Beschäftigten. Die Diskussion von Konsequenzen für die Arbeitsgestaltung und Personalpolitik hat demgegenüber erst ansatzweise begonnen. ${ }^{18}$

Vor diesem Hintergrund seien im Folgenden exemplarisch einige Problemfelder und Perspektiven benannt, um zu verdeutlichen, in welcher Weise sich durch den Blick auf Interaktionsarbeit neue Herausforderungen für die Arbeitspolitik ergeben, die in weiteren Diskussionen und Untersuchungen aufzugreifen, zu vertiefen und weiterzuführen wären. ${ }^{19}$

(1) Integration von Arbeitsgestaltung und Produktgestaltung - Balance zwischen den Interessen der Arbeitenden und den Interessen von Kunden und Klienten: Bei Interaktionsarbeit hat die Berücksichtigung der Interessen der Beschäftigten immer auch Auswirkungen auf Kunden und Klienten, und umgekehrt hat die Orientierung an Kundenwünschen unmittelbar Auswirkungen auf die Beschäftigten. Daraus können sich für die Arbeitsgestaltung positive Effekte ergeben, wenn zur Verbesserung der Qualität der Dienstleistung qualifizierte und selbstverantwortliche Tätigkeiten geschaffen werden. Es können aber ebenso weitreichende Konflikte entstehen. Ein Beispiel hierfür ist die Auseinandersetzung mit der Regelung der Arbeitszeit. Bei Maßnahmen, durch die Belastungen reduziert werden, aber zugleich die Kundenzufriedenheit beeinträchtigt wird, besteht die Gefahr, dass die positiven Effekte für die Beschäftigten neutralisiert werden. So können die Beschäftigten durch die Unzufriedenheit der Kunden in neuer Weise belastet und in der Verwirklichung eigener Ansprüche an die Arbeit eingeschränkt werden. Ein Beispiel für ein solches Konfliktverhältnis ist die „Ruhigstellung“von Patienten in der Pflege. Hierdurch kann die Arbeit erleichtert werden, zugleich wird damit aber nicht nur die Qualität der Pflege beeinträchtigt, sondern auch für die Pflegekräfte die Sinnhaftigkeit ihrer Arbeit. Die Arbeitsgestaltung beeinflusst somit immer auch die Produktgestaltung und umgekehrt hat die Produktgestaltung unmittelbar Auswirkungen auf die Arbeitsgestaltung. Maßnahmen zum Schutz und der Förderung der Gesundheit bis hin zur Förderung der Entwicklung individueller Fähigkeiten und Persönlichkeit müssen daher so ausgelegt sein, dass eine Balance zwischen Beschäftigten und Kundeninte- ressen sowie deren wechselseitige Ergänzung und Verstärkung erreicht wird.

(2) Grenzen der Standardisierung - Besondere Belastungen und kontraproduktive Effekte tayloristischer Rationalisierung: Die tayloristische Rationalisierung negiert wesentliche Elemente der Interaktionsarbeit. Im Besonderen betrifft dies die strukturellen Unbestimmtheiten und Unwägbarkeiten in der Arbeit mit Kunden und Klienten. Durch die Standardisierung von Arbeitsabläufen und Zeitvorgaben werden zusätzlich zu bekannten Belastungen restriktiver Arbeit das notwendige situative Handeln behindert und die Qualität der Dienstleistung beeinträchtigt sowie auch zusätzliche Kosten erzeugt - etwa durch Reklamationen oder die Behinderung des Vollzugs der Dienstleistung durch Kunden und Klienten. ${ }^{20}$ Auch bei ,einfacher“ Arbeit wie in Fast-Food-Restaurants sind daher Handlungsspielräume für selbst gesteuertes Handeln notwendig. Des Weiteren sind die Beeinflussung der Gefühle von Kunden und Klienten sowie der Umgang mit den eigenen Emotionen besondere Anforderungen, die auch bei hoch standardisierter Dienstleistungsarbeit wie dem Verkauf in Fast-Food-Restaurants auftreten. Die gängige Bezeichnung solcher Dienstleistungen als „einfache“ Arbeit blendet jedoch solche Anforderungen weitgehend aus.

15 Siehe in dieser Perspektive zum Einfluss von Kunden und Klienten Voswinkel/Korzekwa (2005); Leidner (1996) sowie Frenkel et al. (1999).

16 Beispiel hierfür sind besondere Arbeitszeitregelungen, aber auch Trainings zur Schulung sozialer Kompetenzen und Kommunikationsfähigkeit.

17 Siehe hierzu die Hinweise und Literaturverweise bei der vorangehenden Darstellung der Merkmale von Interaktionsarbeit, insbesondere zum Umgang mit eigenen Gefühlen und Beeinflussung der Gefühle von Kunden und Klienten. Zu ergänzen sind hier auch Untersuchungen zu Burn-out infolge des subjektiven Involvements insbesondere bei sozialen Berufen. Einen Überblick gibt Moosbrugger (2008).

18 Richtungsweisend hierfür sind z. B. Arbeiten von Hacker (2009); Weishaupt (2006); Weishaupt et al. (2006); Büssing/Glaser (2003). Vgl. auch Ernst/Kopp (2011, S. 266f.).

19 Für Anregungen und Diskussion danke ich Ursula Stöger.

20 Das Konzept der Interaktionsarbeit bestärkt damit Argumente, die auch schon früher gegen die tayloristische Arbeitsorganisation bei Dienstleistungen unter Bezug auf die Qualität des „Produkts“ vorgebracht wurden (vgl. Oberbeck 1997, S. 145; Schlesinger/Heskett 1991). Siehe zu Ineffizienz und zusätzlichen Kosten speziell am Beispiel der Pflege: Böhle (1999). 
(3) Ambivalenzen qualifizierter und selbstverantwortlicher Arbeit-Abgrenzung gegenüber externen Anforderungen und Vertrauen: Neue Strategien der Rationalisierung richten sich verstärkt auf die Nutzung subjektiver Potenziale. In der Arbeitsforschung wird dies als „Subjektivierung von Arbeit" bezeichnet (Moldaschl/Voß2003). Dabei geraten auch neue Belastungen und Ambivalenzen selbstverantwortlicher Arbeit in den Blick. Bei Interaktionsarbeit beinhalten ein breites Aufgabenspektrum und Selbstverantwortung besondere Risiken. Zum einen besteht nicht nur - wie auch bei sonstiger Arbeit - die Gefahr der Selbstüberforderung und Selbstrationalisierung, sondern auch die, durch die Anforderung von Kunden und Klienten „aufgesaugt“ zu werden. Bei der Arbeitsgestaltung wäre es daher notwendig, zugleich auch Möglichkeiten der Abgrenzung und des Rückzugs für die Beschäftigten zu schaffen. Ein Beispiel hierfür ist die gezielte Kombination von Interaktionsarbeit mit Aufgaben, bei denen die Arbeit mit materiellen und immateriellen Objekten im Vordergrund steht. Dabei ist es jedoch notwendig, dass solche Tätigkeiten flexibel wahrgenommen und situativ an die Interaktionsarbeit angepasst werden können. Erforderlich sind dementsprechend zeitliche Puffer sowie Möglichkeiten der Selbststeuerung und Kontrolle durch die Arbeitenden. Zum anderen zielen Unternehmen zunehmend darauf ab, qualifizierte und selbstverantwortliche Arbeit in neuer Weise zu steuern und zu kontrollieren. In der Arbeitsforschung wird dies als Vermarktlichung und indirekte Steuerung bezeichnet. ${ }^{21}$ Ein wesentliches Instrument ist hierfür die Formalisierung und $\mathrm{Ob}$ jektivierung des Arbeitshandelns. Seinen Ausdruck findet dies in Anforderungen an die Dokumentation sowie die Steuerung des Arbeitshandels durch Kennzahlen, Best-Practice-Modelle und formalisierte Verfahren (vgl. Böhle et al. 2011). Das bei Interaktionsarbeit notwendige situative Handeln lässt sich jedoch nur begrenzt formalisieren und objektivieren. So entsteht auch bei qualifizierter, selbstverantwortlicher Arbeit die Gefahr einer Behinderung notwendiger Arbeitsleistung und Gefährdung der Qualität der Dienstleistung. Interaktionsarbeit erfordert daher nicht nur Handlungsspielräume, sondern auch die Anerkennung informeller Arbeitspraktiken und Erfahrungswissens sowie hierauf bezogenes Vertrauen statt Kontrolle.
(4) Anerkennung besonderer Leistungen Leistungsbeurteilung und Personaleinsatz: Die geschilderten Merkmale und Anforderungen bei Interaktionsarbeit tauchen bisher bei der offiziellen Beschreibung von Arbeitsaufgaben und erforderlichen Qualifikationen kaum auf. Sie sind in der Praxis notwendig und werden erwartet, haben aber primär den Charakter von „tacit skills“, das heißt, sie werden stillschweigend gefordert und genutzt, nicht aber honoriert. Notwendig wäre demgegenüber die explizite Nennung und Anerkennung, sodass die Möglichkeit besteht, sie in die Auseinandersetzung über die Leistungsbeurteilung und Entlohnung einzubeziehen. Da solche Leistungen zu großen Teilen nur schwer durch formalisierbare Kriterien erfasst und beurteilt werden können, ist die Entwicklung hierfür geeigneter Verfahren und Instrumente der Leistungsbeurteilung eine besondere Herausforderung an die Arbeitspolitik.

(5) Lernen im Prozess der Arbeit-Entwicklung von Kompetenzen für Interaktionsarbeit: ${ }^{22}$ Obwohl Tätigkeiten im Dienstleistungsbereich bei der Neuordnung von Ausbildungsberufen und der beruflichen Bildung zunehmend beachtet werden, wurden bisher die für Interaktionsarbeit notwendigen Kompetenzen nur begrenzt beachtet. Am ehesten ist dies bei unmittelbar personenbezogenen Dienstleistungen wie der Pflege der Fall. Der Schwerpunkt liegt dabei auf dem Umgang mit eigenen Gefühlen und der Beeinflussung der Gefühle von Patienten. Zumeist beschränkt sich in anderen Bereichen der Bezug auf die Arbeit mit und an Menschen auf allgemeine Schlüsselqualifikationen wie „soziale Kompetenz“, „Dialogfähigkeit“ und „kommunikative Kompetenzen“ oder spezielle Trainings etwa zur Führung von Verkaufsgesprächen. ${ }^{23}$ Im Unterschied $\mathrm{zu}$ allgemeinen Schulungen von sozialer Kompetenz und Kommunikationsfähigkeit ist bei der Interaktionsarbeit vor allem die jeweils fach- und bereichsbezogene Ausformung der Interaktion und damit die unmittelbare Verknüpfung mit den sachlichen Inhalten der Arbeit bedeutsam. Dies gilt speziell für subjektivierendes Handeln, das sich auf den sachlich-funktionalen Inhalt der Dienstleistung bezieht. Des Weiteren erfordert die Entwicklung von Kompetenzen für Interaktionsarbeit vor allem ein Lernen am „Gegenstand“. Eine praktische erfahrungsbezogene Aus- bildung sowie ein kontinuierliches Lernen im Prozess der Arbeit sind unerlässlich. Dies vor allem auch deshalb, weil nicht nur - wie bei sonstiger Arbeit - die Inhalte von Dienstleistungen laufend Veränderungen unterliegen, sondern auch Bedürfnisse und das Verhalten von Kunden und Klienten in hohem Maße sozio-kulturellen Einflüssen und Veränderungen sowie kulturellen, schicht- und milieuspezifischen Differenzierungen ausgesetzt sind. Eine wichtige Voraussetzung zur Entwicklung von Kompetenzen für Interaktionsarbeit ist da aber eine hierauf bezogene lernförderliche Arbeitsgestaltung. ${ }^{24}$

(6) Technik nicht als Ersetzung, sondern als Unterstützung von Interaktionsarbeit: Die Erkenntnisse zu Interaktionsarbeit weisen darauf hin, dass es kaum möglich ist, die Arbeit mit Kunden und Klienten zu automatisieren und zugleich kundenorientierte Dienstleistungen zu gewährleisten. Welche Anforderungen sich daraus an technische Entwicklungen ergeben, müsste in weiteren Diskussionen näher geklärt werden. Weiterführend erscheint hier ein Verständnis von Technik als „Werkzeug“ zur Unterstützung von Interaktionsarbeit. Dabei gilt es zu beachten - im Unterschied zur derzeit vorherrschenden Logik von Informations- und Kommunikationstechnologien -, die Möglichkeit für informelle Arbeitspraktiken und implizites Erfahrungswissen nicht einzuschränken, sondern aufrechtzuerhalten und ggf. zu erweitern. ${ }^{25}$

In einer weitergehenden Perspektive wäre schließlich zu prüfen, in welcher Weise sich aus einer Arbeitspolitik für Interaktionsarbeit auch neue Anregungen und Impulse für eine humane Arbeitsgestaltung von Arbeit insgesamt ergeben.

21 Siehe hierzu allgemein Sauer (2005), Moldasch (1998) sowie speziell unter Bezug auf Dienstleistungen Lehndorff/Voss-Dahm (2006).

22 Der Begriff Kompetenzen bezieht sich im Folgenden sowohl auf sachlich-funktionale Qualifikationen als auch auf Handlungsdispositionen.

23 Siehe hierzu ausführlicher den Überblick bei Brater/Rudolf (2006).

24 Siehe hierzu allgemein insbesondere Bauer et al. 2004 sowie unter Bezug auf Interaktionsarbeit Brater/Rudolf 2006.

25 Siehe in dieser Perspektive grundlegend zu den negativen Auswirkungen von Informations- und Kommunikationstechnologien auf informelle Arbeitspraktiken und Erfahrungswissen Böhle (2001). 
Badura, B./Gross, P. (1976): Sozialpolitische Perspektiven, München Baethge, M./Wilkens, I. (2001): Die große Hoffnung für das 21. Jahrhundert?, Opladen

Bauer, H. G./Brater, M./Büchele, U./Dahlem, H./Maurus, A./

Munz, C. (2004): Lernen im Arbeitsalltag, Bielefeld

Böhle, F. (1999): Nicht nur mehr Qualität, sondern auch höhere Effizienz, in: Zeitschrift für Arbeitswissenschaft 53 (3), S. 174-181

Böhle, F. (2001): Alternativen in der Technikentwicklung - nicht nur die Organisation, sondern auch die Technik entscheidet über die "Zukunft der Arbeit“, in: Weber, W. G./Wehner, T. (Hrsg.): Erfahrungsorientierte Handlungsorganisationen, Zürich, S. 187-214

Böhle, F. (2010): Arbeit als Handeln, in: Böhle, F./Voß, G. G./Wachtler, G. (Hrsg.): Handbuch Arbeitssoziologie, Wiesbaden, S. 151-176

Böhle, F./Glaser, J. (Hrsg.) (2006): Arbeit in der Interaktion - Interaktion als Arbeit, Wiesbaden

Böhle, F./Pfeiffer, S./Porschen, S./Sevsay-Tegethoff, N. (2011):

Herrschaft durch Objektivierung, in: Bonß, W./Lau, C. (Hrsg.):

Wandel und Herrschaft in der Zweiten Moderne, Weilerswist/Velbrück, S. 244-283

Böhle, F./Pfeiffer, S./Sevsay-Tegethoff, N. (Hrsg.) (2004): Die Bewältigung des Unplanbaren, Wiesbaden

Brater, M./Rudolf, P. (2006): Qualifizierung für Interaktionsarbeit - ein Literaturbericht, in: Böhle, F./Glaser, J. (Hrsg.): Arbeit in der Interaktion. Interaktion als Arbeit, Wiesbaden, S. 261-308

Brucks, U. (1999): Gefühlsarbeit. Versuch einer Begriffsklärung, in: Zeitschrift für Arbeitswissenschaft 53 (3), S. 182-186

Büssing, A./Glaser, J. (2003): Arbeitsbelastungen, Burnout und Interaktionsstress im Zuge der Reorganisation des Pflegesystems, in: Büssing, A./Glaser, J. (Hrsg.): Dienstleistungsqualität und Qualität des Arbeitslebens im Krankenhaus, Göttingen, S. 101-129

Dunkel, W. (1988): Wenn Gefühle zum Arbeitsgegenstand werden, in: Soziale Welt 39 (1), S. 66-85

Dunkel, W. (2006): Interaktionsarbeit im Friseurhandwerk - Arbeit am Menschen und Arbeit am Gegenstand, in: Böhle, F./Glaser, J. (Hrsg.): Arbeit in der Interaktion - Interaktion als Arbeit, Wiesbaden, S. 219-234

Dunkel, W./Voss, G. (2004): Dienstleistung als Interaktion, München/ Mering

Dunkel, W./Weihrich, M. (2006): Interaktive Arbeit. Ein Konzept zur Entschlüsselung personenbezogener Dienstleistungsarbeit, in: Dunkel, W./Sauer, D. (Hrsg.): Von der Allgegenwart der verschwindenden Arbeit, Berlin, S. 67-82

Dunkel, W./Weihrich, M. (2010): Arbeit als Interaktion, in: Böhle, F./ Voß, G./Wachtler, G. (Hrsg.): Handbuch Arbeitssoziologie, Wiesbaden, S. $177-200$

Ernst, G./Kopp, I. (2011): Interaktionsarbeit als zentrales Element der Dienstleistungsinnovation, in: Schröder, L./Urban, H.-J. (Hrsg.):

Gute Arbeit, Frankfurt a. M., S. 261-276

Frenkel, S. J./Korczynski, M./Shire, K. A./Tam, M. (1999): On the Front Line. Organization of Work in the Information Economy, Ithaca

Giesenbauer, B./Glaser, J. (2006): Emotionsarbeit und Gefühlsarbeit in der Pflege - Beeinflussung fremder und eigener Gefühle, in: Böhle, F./Glaser, J. (Hrsg.): Arbeit in der Interaktion - Interaktion als Arbeit, Wiesbaden, S. 59-84

Hacker, W. (2005): Allgemeine Arbeitspsychologie, Bern
Hacker, W. (2009): Arbeitsgegenstand Mensch: Psychologie dialogischinteraktiver Erwerbsarbeit, Lengerich

Heidling, E./Böhle, F./Habler, T. (2010): Produktion mit Dienstleistung. Integration als Zukunftschance, München/Mering

Hochschild, A. R. (1979): Emotion Work, Feeling, Rules and Social Structure, in: American Journal of Sociology 85 (3), S. 551-575 Hochschild, A. R. (1983): The Managed Heart, Berkeley Holtgrewe, U. (2001): Organisationsdilemmata und Kommunikationsarbeit, in: Matuschek I./Henninger, A./Kleemann, F. (Hrsg.): Neue Medien im Arbeitsalltag, Wiesbaden, S. 55-70

Kleemann, F./Matuschek, I. (2003): Immer Anschluss unter dieser Nummer, Berlin

Koch, V. (2010): Interaktionsarbeit bei produktbegleitenden Dienstleistungen, Wiesbaden

Lehndorff, S./Voss-Dahm, D. (2006): Kunden, Kennziffern und Konkurrenz, in: Lehndorff, S. (Hrsg.): Das Politische in der Arbeitspolitik, Berlin

Leidner, R. (1996): Re-thinking Questions of Control. Lessons from MacDonald's, in: MacDonald, C. L./Sirani, C. (Hrsg.): Working in the Service Society, Philadelphia

Moldaschl, M. (1998): Internalisierung des Marktes, in: Institut für Sozialwissenschaftliche Forschung München (ISF) et al. (Hrsg.): Jahrbuch Sozialwissenschaftliche Technikberichterstattung 1997, Berlin, S. 197-250 Moldaschl, M./Voß G. G. (2003): Subjektivierung der Arbeit, München/Mering

Moosbrugger, J. (2008): Subjektivierung von Arbeit: Freiwillige Selbstausbeutung, Wiesbaden

Nerdinger, F. W. (1994): Zur Psychologie der Dienstleistung, Stuttgart Oberbeck, H. (1997): Die Entwicklung der Arbeit in der Dienstleistungsgesellschaft, in: Altvater, E./Haug, F./Negt, O. (Hrsg.): TurboKapitalismus, Hamburg

Sauer, D. (2005): Arbeit im Übergang, Hamburg

Schlesinger, L. A./Heskett, J. L. (1991): The Service-Driven Service Company, in: Harvard Business Review 69 (5), S. 71-81

Spath, D./ Ganz, W. (Hrsg.) (2009): Die Zukunft der Dienstleistungswirtschaft, München

Strauss, A./Fagerhaug, S./Suczek, B./Wiener, C. (1980): Gefühlsarbeit, in: Kölner Zeitschrift für Soziologie und Sozialpsychologie 32 (4), S. 629-651

Vereinte Dienstleistungsgewerkschaft (ver.di), Bereich Innovation und gute Arbeit (Hrsg.) (2011): Arbeit mit Kunden, Patienten, Klienten, Hamburg

Voß, G. G./Rieder, K. (2005): Der Arbeitende Kunde. Frankfurt a. M./ New York

Voswinkel, S./Korzekwa, A. (2005): Welche Kundenorientierung?, Berlin Weihrich, M./Dunkel, W. (2003): Abstimmungsprobleme in

Dienstleistungsbeziehungen, in: Kölner Zeitschrift für Soziologie und Sozialpsychologie 55 (4), S. 758-781

Weishaupt, S. (2006): Subjektivierendes Arbeitshandeln in der Altenpflege - Die Interaktion mit dem Körper, in: Böhle, F./Glaser, J. (Hrsg.): Arbeit in der Interaktion - Interaktion als Arbeit, Wiesbaden

Weishaupt, S./Bolte, A./Iwer, F. (2006): Förderung von Interaktionsarbeit im Produktmanagement der Softwarebranche, in: Böhle, F./Glaser, J. (Hrsg.): Arbeit in der Interaktion - Interaktion als Arbeit, Wiesbaden Zapf, D. (2002): Emotion Work and Psychological Well-Being, in: Human Resource Management Review 12 (2), S. 237-268 